\title{
Nonlinear PD plus sliding mode control with application to a parallel delta robot
}

\author{
Chems Eddine Boudjedir ${ }^{*}$, Djamel Boukhetala*, Mohamed Bouri ${ }^{* *}$
}

\begin{abstract}
In this paper, a hybrid nonlinear proportional-derivative-sliding mode controller (NPD-SMC) is developed for the trajectory tracking of robot manipulators. The proposed controller combines the advantage of the easy implementation of NPD control and the robustness of SMC. The gains of PD control are tuned on-line in order to increase the convergence rate, whereas the SMC term is introduced to reject the external disturbances without requiring to know the system dynamics. The stability of the NPD-SMC is proved using Lyaponuv theorem, and it is demonstrated that the tracking error and the tracking error rate converge asymptotically to zero. Experiments are carried out on the parallel Delta robot to illustrate the effectiveness and robustness of the proposed approach. It is also shown the superiority of the NPD-SMC control over the NPD control and PD-SMC control.
\end{abstract}

K e y w ord s: nonlinear PD, sliding mode control, stability, delta robot, trajectory tracking

\section{Introduction}

The utilisation of robot manipulators has been increased during the last decades thanks to their high quality of manufacture. Meanwhile, much research has been conducted to solve the trajectory tracking problem. Usually, the conventional PD/PID controllers are used due to their easy implementation and acceptable performances. However, PD/PID failed to track the desired trajectory for high-dynamic movement where strong nonlinearity and coupling have an important effect. To increase the robustness of PD/PID, nonlinear gains tuned on-line using the tracking error and its time derivative were introduced instead of the fixed gain $[1,2]$. Nevertheless, the robustness range of NPD/NPID is limited by controller structure, high dynamic movement, and external disturbances. To overcome this shortcoming, many control strategies have been proposed [3-7]. Fuzzy logic control was implemented successfully in some robots [8]. However, the choice of the linguistic rules and guaranteeing the system stability still remain a challenging issue. Moreover, the industrial implementation is not an easy task. In many adaptive control [6], the dynamic model is supposed a premultiplied of two separate linear unknown parameters with known nonlinear functions. However, finding this separation cannot always be systematic, especially for complex systems. Sliding mode control is knowing by its ability to decrease the tracking error with a wide range of robustness for nonlinear systems subject to model uncertain and disturbances. The drawback of SMC is that require to know the system dynamic, as a consequence, the implementation can be challenging and priced. Another issue represents in the chattering phenomena which can damage the actuators. In order to benefit from the SMC robustness and the easy implementation of PD control, a hybrid PD plus SMC (PD-SMC) schemes have been proposed in some works. In [9] the authors developed PD-SMC to solve the trajectory tracking problem of robot manipulators with an application to a two-link SCARA robot, where PD control is applied in the reaching phase and the semicontinuous sliding mode control is applied in the sliding mode phase. However, semicontinuous sliding mode phase requires the knowledge of the exact dynamic which could be a challenging issue. In [10] an adaptive sliding mode control with PID tuning was proposed for uncertain systems. However, the controller is complex due to the requirement of the dynamic model. Moreover, there is no experimental validation. In [11], a PD-SMC was developed for trajectory tracking control of robot manipulators where some tuning rules were also discussed through simulation. However, the inertia matrix, the Coriolis and centrifuge matrix and the gravity vector were considered constant, as a consequence, the convergence analysis cannot be generalised to the other robot manipulators such as the parallel Delta robot.

Delta robot is a parallel manipulator invented by $\mathrm{R}$. Clavel [12] in order to execute pick and place operations at a high dynamic movement. Many control strategies have been implemented on the Delta robot such as, PD plus feedforward control [13], a linear robust Hinfinity control that was synthesised around an operating point [14] and an adaptive control that was developed for uncertain model in [15]. However, the drawback of the above-mentioned works is that knowing the exact dynamic model is crucial to satisfy the required performances.

* Laboratory of Process Control (LCP), Ecole Nationale Polytechnique (ENP), 10 Rue des Freres OUDEK, El-Harrach 16200, Algiers, Algeria, chemseddine.boudjedir@g.enp.edu.dz, djamel.boukhetala@g.enp.edu.dz, ** Laboratory of Robotic Systems (LSRO), Ecole Polytechnique Fédérale de Lausanne (EPFL), Station 9, Lausanne CH-1015, Switzerland, mohamed.bouri@epfl.ch 
This paper proposes a NPD-SMC to solve the trajectory tracking problem of robot manipulators. In the proposed controller a nonlinear PD control replaced the fixed gains $\mathrm{PD}$ control in order to increase the convergence rate and a SMC is introduced in order to increase the robustness range and to reject the disturbances. Unlike $[9,11]$, the proportional and derivative gains are adapted on-line using the tracking error and its time derivative respectively. Unlike, [11] the dynamic model matrices are not constant but depend on the joints coordinate. Convergence analysis of the proposed approach is driven using Lyapunov technique. Experiments are carried out on a parallel Delta robot and a comparative study between the NPD, the PD-SMC and the NPD-SMC is provided in order to show the effectiveness and the high tracking performances of this latter.

Throughout this paper, the norm of vector $x$ is defined as $\|x\|=\sqrt{x^{\top} x}$, and the norm of matrix $A$ is defined as follows: $\|A\|=\sqrt{\lambda_{\max }\left(A^{\top} A\right)}$, where $\lambda_{\max }(\cdot)$ indicate the maximum eigenvalue of matrix $(\cdot)$.

\section{Problem formulation}

Consider a rigid robot manipulator with a n-degree of freedom (DOF) described by following dynamic equation

$$
M(q) \ddot{q}+C(q, \dot{q}) \dot{q}+G(q)+w(t)=\tau
$$

where $q \in \mathbb{R}^{n}$ is the generalised joint vector, $M(q) \in$ $\mathbb{R}^{n \times n}$ is the inertia matrix, $C(q, \dot{q}) \dot{q} \in \mathbb{R}^{n}$ is a vector resulting from Coriolis and centrifugal forces, $G(q) \in \mathbb{R}^{n}$ is the gravity torque vector, $\tau \in \mathbb{R}^{n}$ is the control input vector containing the torques to be applied at each joint and $w(t) \in \mathbb{R}^{n}$ is the vector contain external disturbances.

The actual dynamics (1) can be written using the nominal model in the following formulation

$$
M_{n}(q) \ddot{q}+C_{n}(q, \dot{q}) \dot{q}+G_{n}(q)+d(t)=\tau .
$$

The expression of the lumped disturbances $d(t)$ is given as follows

$$
d(t)=\Delta M(q) \ddot{q}+\Delta C(q, \dot{q}) \dot{q}+\Delta G(q)+w(t)
$$

where $\Delta$ indicates the modelling error.

The following properties, common to robot manipulators are considered [16-18]:

(P1) The inertia matrix $M_{n}(q)$ is symmetric, positive and satisfies

$$
0<\beta_{1} \leq\left\|M_{n}(q)\right\| \leq \beta_{2}
$$

where $\beta_{1}$, and $\beta_{2}$ are known positive constants.

(P2) The norm of the Coriolis-centrifugal matrix and the gravity vector is bounded as follows

$$
\left\|C_{n}(q, \dot{q})\right\| \leq K_{c}\|\dot{q}\|,\left\|G_{n}(q)\right\| \leq K_{G}, \forall q, \dot{q} \in \mathbb{R}^{n}
$$

where $K_{c}$ and $K_{G}$ are a known positive constants.

(P3) The matrix $\dot{M}(q)-2 C(q, \dot{q})$ is skew symmetric, hence

$$
q^{\top}(\dot{M}(q)-2 C(q, \dot{q})) q=0 \quad \forall q \in \mathbb{R}^{n} .
$$

Throughout this paper, the following reasonable assumptions are made:
Assumption 1. The robot velocity is bounded by a known constant $V_{m}$, such that

$$
\|\dot{q}\| \leq V_{m}
$$

Assumption 2. The lumped disturbances are bounded by a constant $l_{d}$, such that

$$
\|d(t)\| \leq l_{d}
$$

Assumption 3. The desired trajectory $q_{d}(t)$ up to its $n$th derivative are continuous, bounded and available, for instance

$$
\left\|\ddot{q}_{d}(t)+\lambda \dot{q}_{d}(t)\right\| \leq \alpha
$$

where $\alpha$ and $\lambda$ are positive constants.

$\mathrm{R}$ e mark 1 . Assumption 1 is very common in real time application where many real systems are subjected to state and input constraints due to the safety requirements and the physical limitations of the actuators. As a result, the velocity joints can be bounded by a known constant. This assumption has been considered in many works such as $[19,20]$.

Remark 2. Assumption 2 is very reasonable for real-time application where due to the safety requirements and the tracking error tolerance, the disturbances are always bounded. This assumption has been considered in many real-time applications such as mobile wheeled inverted pendulum [21].

The control objective of this paper is to design a robust control law for system (2) such that the tracking position error and the tracking velocity error can converge asymptotically to zero with a simple implementation scheme.

\section{Proposed NPD-SMC}

\subsection{Controller design}

The proposed control law is expressed as

$$
\tau(t)=K_{p}(\tilde{q}) \tilde{q}+K_{d}(\dot{\tilde{q}}) \dot{\tilde{q}}+H \operatorname{sgn}(\dot{\tilde{q}}+\lambda \tilde{q})
$$

and

$$
\begin{aligned}
& K_{p}(\tilde{q})=\operatorname{diag}\left\{K_{p_{1}}\left(\tilde{q}_{1}\right), \ldots, K_{p_{n}}\left(\tilde{q}_{n}\right)\right\} \\
& K_{d}(\dot{\tilde{q}})=\operatorname{diag}\left\{K_{d_{1}}\left(\dot{\tilde{q}}_{1}\right), \ldots, K_{d_{n}}\left(\dot{\tilde{q}}_{n}\right)\right\}
\end{aligned}
$$


where

$$
\begin{gathered}
K_{p_{i}}\left(\tilde{q}_{i}\right)= \begin{cases}K_{p 0}\left|\tilde{q}_{i}\right|^{\alpha_{1}-1}, & \left|\tilde{q}_{i}\right|>\delta_{1}, i=1, \ldots, n, \\
K_{p 0} \delta_{1}^{\alpha_{1}-1}, & \left|\tilde{q}_{i}\right| \leq \delta_{1}, \\
K_{\mathrm{pmax}}, & K_{p_{i}}\left(\tilde{q}_{i}\right) \geq K_{\mathrm{pmax}}, \\
K_{\mathrm{pmin}}, & K_{p_{i}}\left(\tilde{q}_{i}\right) \leq K_{\mathrm{pmin}},\end{cases} \\
K_{d_{i}}\left(\dot{\tilde{q}}_{i}\right)= \begin{cases}K_{d 0}\left|\dot{\tilde{q}}_{i}\right|^{\alpha_{2}-1}, & \left|\dot{\tilde{q}}_{i}\right|>\delta_{2}, i=1, \ldots, n, \\
K_{d 0} \delta_{2}^{\alpha_{2}-1}, & \left|\dot{\tilde{q}}_{i}\right| \leq \delta_{2}, \\
K_{\mathrm{dmax}}, & K_{d_{i}}\left(\dot{\tilde{q}}_{i}\right) \geq K_{\mathrm{dmax}}, \\
K_{\mathrm{dmin}}, & K_{d_{i}}\left(\dot{\tilde{q}}_{i}\right) \leq K_{\mathrm{dmin}} .\end{cases}
\end{gathered}
$$

Index $i$ indicates the $i$ th row of a vector. The expression of $\tilde{q}$ and $\dot{\tilde{q}}$ is

$$
\tilde{q}=q_{d}-q, \quad \dot{\tilde{q}}=\dot{q}_{d}-\dot{q}
$$

where, $q_{d}$ and $\dot{q}_{d}$ represent the desired joint position and the desired joint velocity respectively. The gains $K_{p 0}$, $K_{\text {pmax }}, K_{\text {pmin }}, K_{d 0}, K_{\mathrm{dmax}}, K_{\mathrm{dmin}}$ and $H$ are positive constant.

Variables $\alpha_{1}$ and $\alpha_{2}$ refer to the nonlinearity, while $\delta_{1}$ and $\delta_{2}$ refer to the threshold of the error and its time derivative respectively.

R e m a r k 3 . The nonlinear gains (13) and (14) indicate that a small gain will be used for a small error and a large gain for a large error. On the other hand, a small gain will be used for a small change of the error a large gain for a large change of the error.

$\mathrm{R}$ e $\mathrm{m}$ ark 4 . As it can be seen from the controller law, a very important aspect of the NPD-SMC reside in its easy implementation and integration in the industrial robots.

\subsection{Convergence analysis}

The convergence of the proposed NPD-SMC is guaranteed by the following theorem.

Theorem. Consider the system (2) under the control law (10), assumption (A1-A3) and satisfies properties (P1-P3). Then the tracking position error and the tracking velocity error converge asymptotically to zero, ie, $\lim _{t \rightarrow \infty} \tilde{q}(t)=\lim _{t \rightarrow \infty} \dot{\tilde{q}}(t)=0$, provided that the control gains are chosen such that

$$
\begin{gathered}
H>\alpha \beta_{2}+K_{c} V_{m}^{2}+\lambda \beta_{2} V_{m}+K_{G}+l_{d}, \\
2 \lambda K_{\mathrm{pmin}}>2 \lambda \mu+2 \lambda^{2} \mu+\lambda K_{\mathrm{dmax}}, \\
2 K_{\mathrm{d} \min }>2 \lambda \mu+2 \mu+\lambda K_{\mathrm{dmax}}
\end{gathered}
$$

where, $\mu=\max \left\|C_{n}(q(t), \dot{q}(t))\right\|$, for all $t \in \mathbb{R}^{+}$.

Pr o of . To simplify the notation, let

$$
\begin{gathered}
M_{n}(q)=M_{n}, \quad C_{n}(q, \dot{q})=C_{n}, \quad G_{n}(q)=G_{n}, \quad d(t)=d \\
K_{p}(\tilde{q}(t))=K_{p}, \quad K_{d}(\dot{\tilde{q}}(t))=K_{d}
\end{gathered}
$$

Define a Lyapunov function as follows

$$
V(\tilde{q}(t), \dot{\tilde{q}}(t))=\frac{1}{2}(\dot{\tilde{q}}+\lambda \tilde{q})^{\top} M_{n}(\dot{\tilde{q}}+\lambda \tilde{q})+\int_{0}^{\tilde{q}} \sigma^{\top} K_{p}(\sigma) d \sigma
$$

Function $\int_{0}^{\tilde{q}} \sigma^{\top} K_{p}(\sigma) d \sigma$ is positive definite as shown in [1]. Also, that above integral is radially unbounded with respect to $\tilde{q}$ and this implies $\int_{0}^{\tilde{q}} \sigma^{\top} K_{p}(\sigma) d \sigma \rightarrow \infty$ as $|\tilde{q}| \rightarrow \infty$. By differentiating $V$ with respect to time, we obtain

$$
\begin{aligned}
\dot{V}=(\dot{\tilde{q}}+\lambda \tilde{q})^{\top} & M_{n}(\ddot{\tilde{q}}+\lambda \dot{\tilde{q}}) \\
& +\frac{1}{2}(\dot{\tilde{q}}+\lambda \tilde{q})^{\top} \dot{M}_{n}(\dot{\tilde{q}}+\lambda \tilde{q})+\tilde{q}^{\top} K_{p}(\tilde{q}) \dot{\tilde{q}}
\end{aligned}
$$

The differentiation of $\int_{0}^{\tilde{q}} \sigma^{\top} K_{p}(\sigma) \mathrm{d} \sigma$ is made via the Leibniz rule for differentiation of integrals.

By substituting (2) into (19), we get

$$
\begin{aligned}
\dot{V}=(\dot{\tilde{q}}+\lambda \tilde{q})^{\top} & M_{n}\left[\ddot{q}_{d}-M_{n}^{-1}\left(\tau-C_{n} \dot{q}-G_{n}-d\right)+\lambda \dot{\tilde{q}}\right] \\
& +\frac{1}{2}(\dot{\tilde{q}}+\lambda \tilde{q})^{\top} \dot{M}_{n}(\dot{\tilde{q}}+\lambda \tilde{q})+\tilde{q}^{\top} K_{p} \dot{\tilde{q}}
\end{aligned}
$$

Using property (P3), (20) becomes

$$
\begin{aligned}
& \dot{V}=(\dot{\tilde{q}}+\lambda \tilde{q})^{\top} M_{n} {\left[\ddot{q}_{d}-M_{n}^{-1}\left(\tau-C_{n} \dot{q}-G_{n}-d\right)+\lambda \dot{\tilde{q}}\right] } \\
&+(\dot{\tilde{q}}+\lambda \tilde{q})^{\top} C_{n}(\dot{\tilde{q}}+\lambda \tilde{q})+\tilde{q}^{\top} K_{p} \dot{\tilde{q}} .
\end{aligned}
$$

Substituting the control law (10) into (21) yields

$$
\begin{aligned}
& \dot{V}=(\dot{\tilde{q}}+\lambda \tilde{q})^{\top}\left[M_{n} \ddot{q}_{d}-K_{p} \tilde{q}-K_{d} \dot{\tilde{q}}-H \operatorname{sgn}(\dot{\tilde{q}}+\lambda \tilde{q})\right. \\
& \left.\quad+C_{n} \dot{q}+G_{n}+d+M_{n} \lambda \dot{\tilde{q}}+C_{n}(\dot{\tilde{q}}+\lambda \tilde{q})\right]+\tilde{q}^{\top} K_{p} \dot{\tilde{q}}
\end{aligned}
$$

Then (22) can be rewritten as

$$
\begin{aligned}
\dot{V}= & (\dot{\tilde{q}}+\lambda \tilde{q})^{\top}\left[M_{n}\left(\ddot{q}_{d}+\lambda \dot{q}_{d}\right)-K_{p} \tilde{q}-K_{d} \dot{\tilde{q}}-H \operatorname{sgn}(\dot{\tilde{q}}+\lambda \tilde{q})\right. \\
& \left.+\left(C_{n}-M_{n} \lambda\right) \dot{q}+G_{n}+d+C_{n}(\dot{\tilde{q}}+\lambda \tilde{q})\right]+\tilde{q}^{\top} K_{p} \dot{\tilde{q}}
\end{aligned}
$$

Therefore

$$
\begin{gathered}
\dot{V} \leq(\dot{\tilde{q}}+\lambda \tilde{q})^{\top}\left(-K_{p} \tilde{q}-K_{d} \dot{\tilde{q}}+C_{n}(\dot{\tilde{q}}+\lambda \tilde{q})\right)+\tilde{q}^{\top} K_{p} \dot{\tilde{q}} \\
+\|\dot{\tilde{q}}+\lambda \tilde{q}\|\left\|M_{n}\left(\ddot{q}_{d}+\lambda \dot{q}_{d}\right)\right\|+\|\dot{\tilde{q}}+\lambda \tilde{q}\|\left\|C_{n} \dot{q}\right\| \\
+\|\dot{\tilde{q}}+\lambda \tilde{q}\|\left\|M_{n} \lambda \dot{q}\right\|+\|\dot{\tilde{q}}+\lambda \tilde{q}\|\left\|G_{n}+d\right\| \\
+\|\dot{\tilde{q}}+\lambda \tilde{q}\|(-H) .
\end{gathered}
$$

We can obtain based on properties (P1-P2) and assumptions $(1-3)$

$$
\begin{gathered}
\dot{V} \leq(\dot{\tilde{q}}+\lambda \tilde{q})^{\top}\left(-K_{p} \tilde{q}-K_{d} \dot{\tilde{q}}+C_{n}(\dot{\tilde{q}}+\lambda \tilde{q})\right)+\tilde{q}^{\top} K_{p} \dot{\tilde{q}}+ \\
\|\dot{\tilde{q}}+\lambda \tilde{q}\|\left(\alpha \beta_{2}+K_{c} V_{m}^{2}+\lambda \beta_{2} V_{m}+K_{G}+l_{d}-H\right)
\end{gathered}
$$




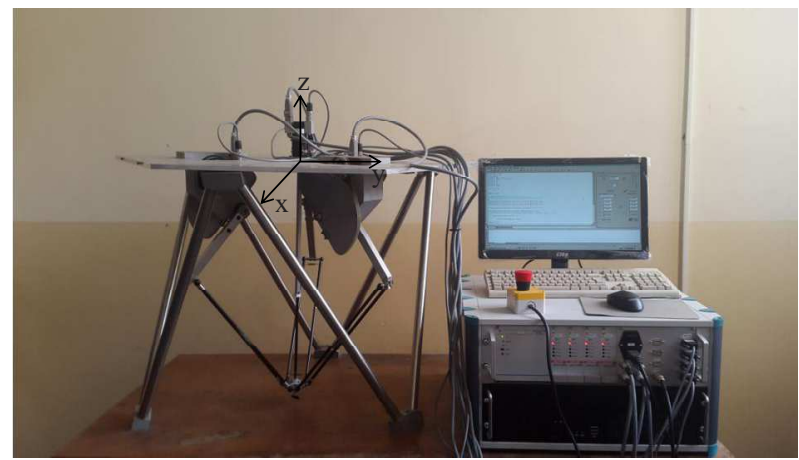

Fig. 1. The delta robot

Table 1. Geometric and dynamic parameters

\begin{tabular}{cll}
\hline Parameter & Description & Value \\
\hline$L_{A}$ & Length of upper arm & $0.380 \mathrm{~m}$ \\
$L_{B}$ & Length of forearm & $0.205 \mathrm{~m}$ \\
$m_{n}$ & Mass of the travelling plate & $0.042 \mathrm{~kg}$ \\
$m_{b r}$ & Mass of the upper arm & $0.098 \mathrm{~kg}$ \\
$m_{f b}$ & Masses of the forearms & $0.028 \mathrm{~kg}$ \\
$m_{c}$ & Mass of the elbow & $0.015 \mathrm{~kg}$ \\
\hline
\end{tabular}

We have

$$
\begin{aligned}
& (\dot{\tilde{q}}+\lambda \tilde{q})^{\top}\left(-K_{p} \tilde{q}-K_{d} \dot{\tilde{q}}+C_{n}(\dot{\tilde{q}}+\lambda \tilde{q})\right)+\tilde{q}^{\top} K_{p} \dot{\tilde{q}} \leq \\
& -\lambda K_{p \min }\|\tilde{q}\|^{2}-K_{d m i n}\|\dot{\tilde{q}}\|^{2}+\|\tilde{q}\|\left\|\lambda K_{d}\right\|\|\dot{\tilde{q}}\|+\lambda^{2} \mu\|\tilde{q}\|^{2} \\
& +\mu\|\dot{\tilde{q}}\|^{2}+2 \lambda \mu\|\tilde{q}\|\|\dot{\tilde{q}}\| .
\end{aligned}
$$

Then, we can obtain

$$
\begin{aligned}
& (\dot{\tilde{q}}+\lambda \tilde{q})^{\top}\left(-K_{p} \tilde{q}-K_{d} \dot{\tilde{q}}+C_{n}(\dot{\tilde{q}}+\lambda \tilde{q})\right)+\tilde{q}^{\top} K_{p} \dot{\tilde{q}} \leq \\
& -\lambda K_{\text {pmin }}\|\tilde{q}\|^{2}-K_{\mathrm{dmin}}\|\dot{\tilde{q}}\|^{2}+\|\tilde{q}\|\left(\lambda K_{\mathrm{dmax}}\right)\|\dot{\tilde{q}}\|+\lambda^{2} \mu\|\tilde{q}\|^{2} \\
& +\mu\|\| \tilde{\tilde{q}}\left\|^{2}+2 \lambda \mu\right\| \tilde{q}\|\| \tilde{\tilde{q}} \|
\end{aligned}
$$

Considering the fact that

$$
\|\tilde{q}\|\|\dot{\tilde{q}}\| \leq \frac{\|\tilde{q}\|^{2}}{2}+\frac{\|\dot{\tilde{q}}\|^{2}}{2}
$$

leads to the following upper bound

$$
\begin{gathered}
(\dot{\tilde{q}}+\lambda \tilde{q})^{\top}\left(-K_{p} \tilde{q}-K_{d} \dot{\tilde{q}}+C_{n}(\dot{\tilde{q}}+\lambda \tilde{q})\right)+\tilde{q}^{\top} K_{p} \dot{\tilde{q}} \leq \\
\left(-\lambda K_{p \min }+\frac{\lambda K_{\text {dmax }}}{2}+\lambda \mu+\lambda^{2} \mu\right)\|\tilde{q}\|^{2} \\
+\left(-K_{\mathrm{d} \min }+\frac{\lambda K_{\mathrm{d} \max }}{2}+\lambda \mu+\mu\right)\|\dot{\tilde{q}}\|^{2} .
\end{gathered}
$$

As a result (25) becomes

$$
\begin{aligned}
\dot{V} \leq \| \dot{\tilde{q}} & +\lambda \tilde{q} \|\left(\alpha \cdot \beta_{2}+K_{c} V_{m}^{2}+\lambda \cdot \beta_{2} \cdot V_{m}+K_{G}+l_{d}-H\right) \\
& +\left(-\lambda K_{\mathrm{pmin}}+\frac{\lambda K_{\mathrm{dmax}}}{2}+\lambda \mu+\lambda^{2} \mu\right)\|\tilde{q}\|^{2} \\
& +\left(-K_{\mathrm{d} \min }+\frac{\lambda K_{\mathrm{d} \max }}{2}+\lambda \mu+\mu\right)\|\dot{\tilde{q}}\|^{2}
\end{aligned}
$$

By using (15), (16) and (17) it is obvious that

$$
\dot{V} \leq 0
$$

Therefore, $\dot{V}$ is negative definite. Since $V$ is positive definite and $\dot{V}$ is negative definite, Lyapunov's method can guarantees that the tracking error and its time derivative are globally uniformly asymptotically stable [22].

We conclude that

$$
\begin{aligned}
& \lim _{t \rightarrow \infty} \dot{\tilde{q}}=0, \\
& \lim _{t \rightarrow \infty} \tilde{q}=0
\end{aligned}
$$

which concludes the proof. As it can be noticed, the convergence analysis indicates that there exist positive gains allow us to obtain the asymptotic convergence.

R e mark 5 . It is worth noting that the sign function used in the proposed control (10) might lead to the chattering phenomenon in control inputs. To reduce this phenomenon in practical applications, saturation function can be introduced instead. As a consequence, the tracking error converges to a domain around zero with a smooth control signal.

\section{Experimental studies}

In this section, we present the experimental results obtained by applying the NPD-SMC on the Delta robot illustrated in Fig. 1.

The Delta robot has 4 DOF, three translations respectively in the directions $x, y$ and $z$ and the rotation $R z$, of the mobile plate around the axis $z$. The inverse dynamic model of the Delta robot is developed based on the principle of virtual work [13], where:

$$
\begin{gathered}
M(q)=I_{b}+m_{n t} J^{\top} J, \quad C(q, \dot{q})=J^{\top} m_{n t} \dot{J}, \\
G(q)=-\tau_{G n}-\tau_{G b} .
\end{gathered}
$$

For the detailed expressions of the Jacobian, $m_{n t}, \tau_{G n}$ and $\tau_{G b}$ please refer to [13]. The parameters of the geometrical and dynamic models are detailed in Table 1.

The experimental implementation is carried out on a Core Duo PC, running at $2,8 \mathrm{GHz}$ under Windows $\mathrm{XP}$ and a real-time extension (RTX) from IntervalZero. The robot actuators are brushed DC motors (minertia motor mini series UGTMEM-03LB2), with a belt-driven transmission of ratio $r_{g}=12$.

The control algorithms were programmed in $\mathrm{C}$ language and execute at a sampling time of $1 \mathrm{~ms}$.

The controller gains are selected as: $K_{p 0}=2.2, K_{d 0}=$ $0.5, K_{\text {pmin }}=1.2, K_{\mathrm{pmax}}=3.0, K_{\mathrm{dmin}}=0.3, K_{\mathrm{dmax}}=$ $0.7, \lambda=0.5, \alpha_{1}=0.4, \alpha_{2}=0.6, \delta_{1}=0.2, \delta_{2}=0.7$, 


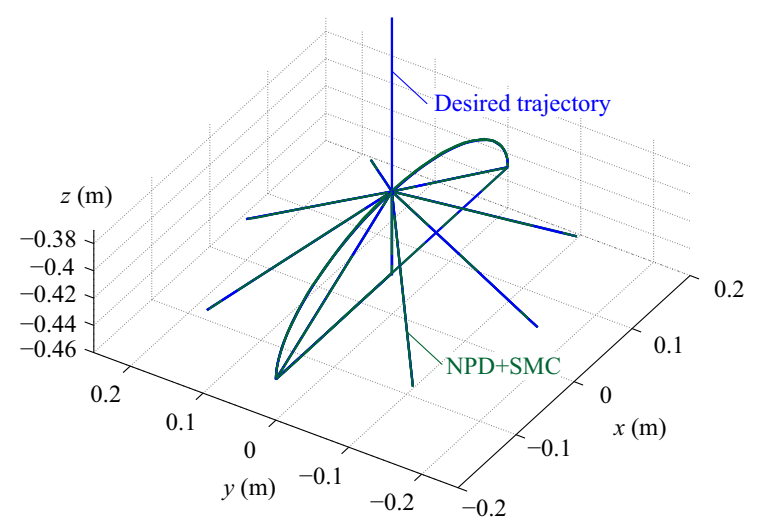

Fig. 2. The operational trajectory tracking under the proposed controlle

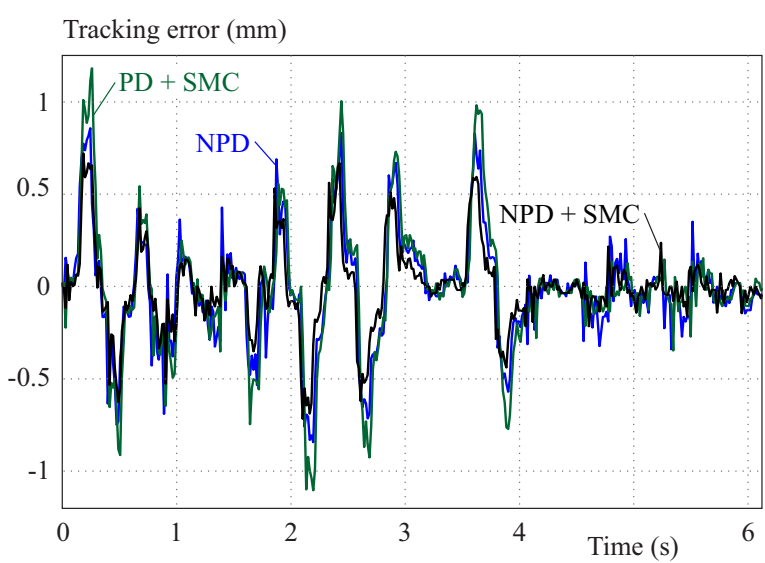

Fig. 4. Experimental tracking error in $y$-axis for case 1 Control torque $(\mathrm{Nm})$

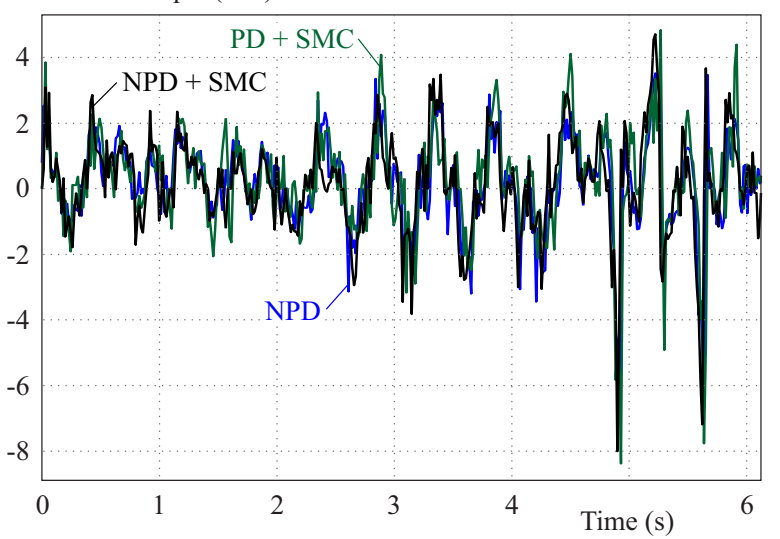

Fig. 6. Experimental torque of joint 1 for case 1

Table 2. Tracking performance for case 1

\begin{tabular}{cccc}
\hline Controller & NPD [1] & PD+SMC [11] & NPD+SMC \\
\hline$R M S E_{x}(\mathrm{~m})$ & $4.3980 \times 10^{-4}$ & $5.5766 \times 10^{-4}$ & $3.7585 \times 10^{-4}$ \\
$R M S E_{y}(\mathrm{~m})$ & $2.9400 \times 10^{-4}$ & $3.5341 \times 10^{-4}$ & $2.2682 \times 10^{-4}$ \\
$R M S E_{z}(\mathrm{~m})$ & $2.9315 \times 10^{-4}$ & $3.6034 \times 10^{-4}$ & $2.9430 \times 10^{-4}$ \\
$R M S E(\mathrm{~m})$ & $6.0481 \times 10^{-4}$ & $7.5214 \times 10^{-4}$ & $5.0485 \times 10^{-4}$ \\
\hline
\end{tabular}

and $H=2$. The gains $K_{p 0}$ and $K_{d 0}$ are selected for the PD-SMC controller.

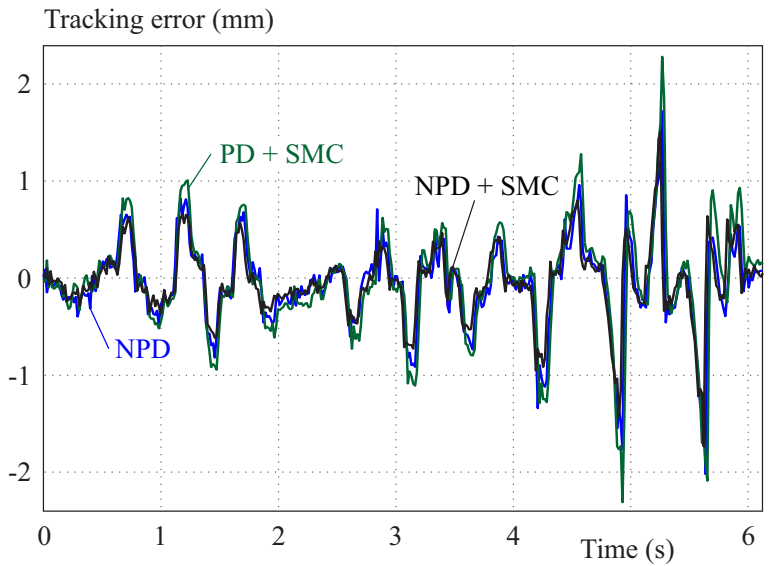

Fig. 3. Experimental tracking error in $x$-axis for case 1

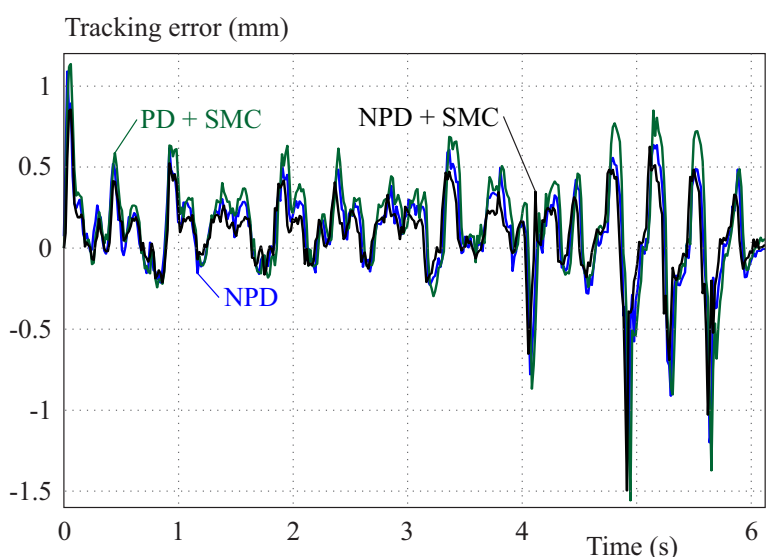

Fig. 5. Experimental tracking error in $z$-axis for case 1

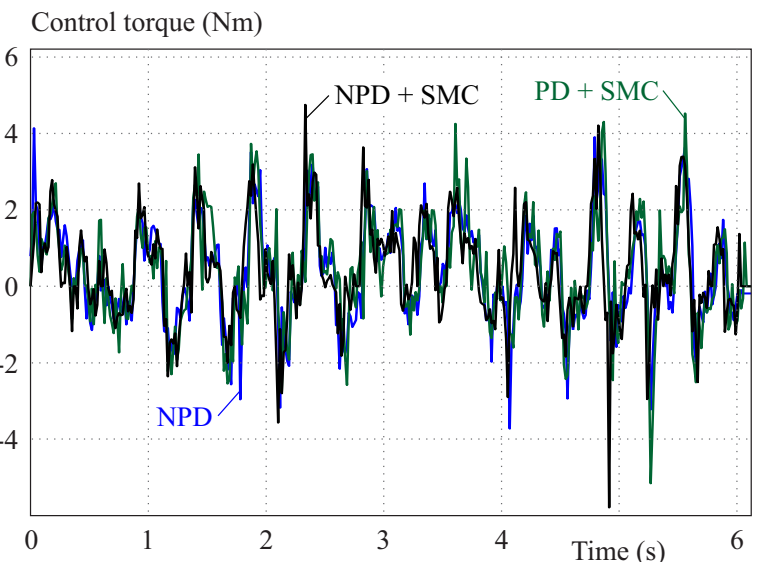

Fig. 7. Experimental torque of joint 2 for case 1

To evaluate the performance of the controllers, the root mean square error (RMSE) of the end-effector position is selected as a performance index. Its expression is given by

$$
R M S E=\sqrt{R M S E_{x}^{2}+R M S E_{y}^{2}+R M S E_{z}^{2}}
$$

with

$$
R M S E_{x}=\sqrt{\frac{1}{n} \sum_{i=1}^{n}\left(x_{i}-x_{d_{i}}\right)^{2}}
$$




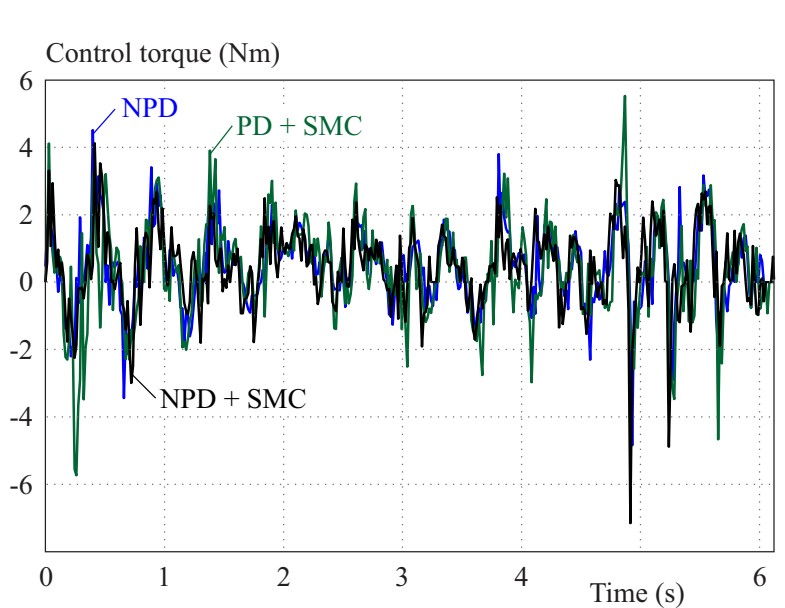

Fig. 8. Experimental torque of joint 3 for case 1

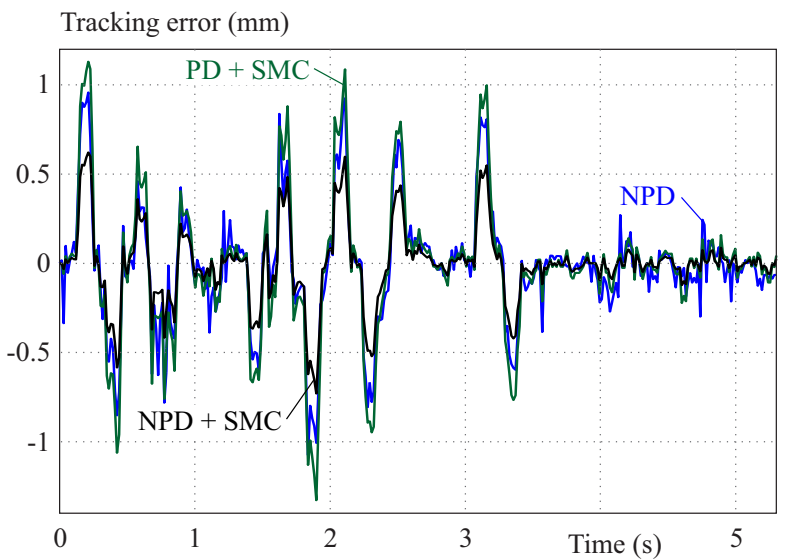

Fig. 10. Experimental tracking error in y-axis for case 2

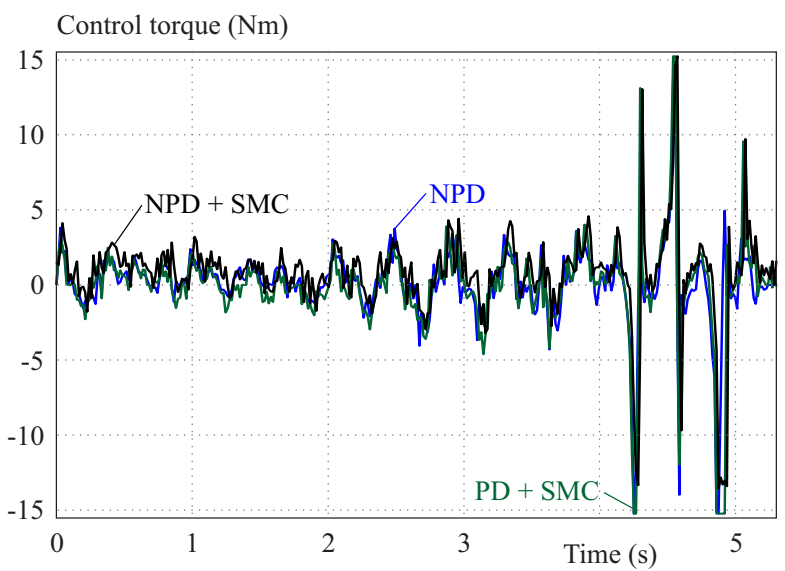

Fig. 12. Experimental torque of joint 1 for case 2

where $x_{d_{i}}$ is the desired trajectory, $x_{i}$ is the actual response, and $n$ is the number of samples.

The desired operational trajectory is a combination of semi-elliptic realised with a parabolic position profile and straight lines as described in Fig. 2, where the starting point is $(0,0,-0.37) \mathrm{m}$ and the ending point is $(0,0,-0.43) \mathrm{m}$. The experiments are given in two cases.

Case 1. This case is considered as a nominal one where the desired trajectory has a maximum velocity of $7.5 \mathrm{~m} / \mathrm{s}$,

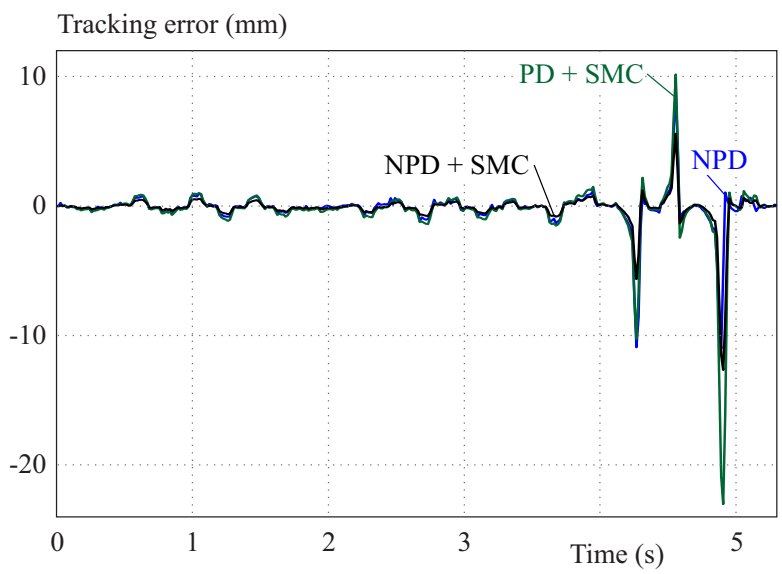

Fig. 9. Experimental tracking error in $x$-axis for case 2

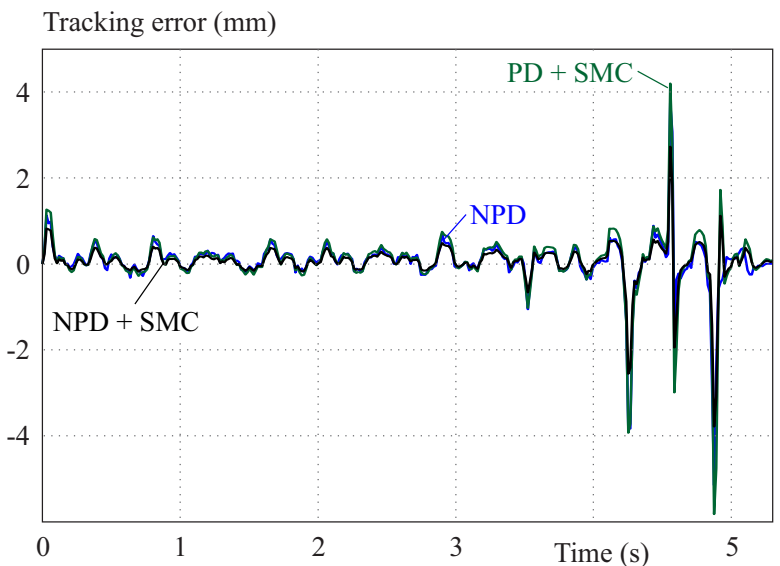

Fig. 11. Experimental tracking error in z-axis for case 2

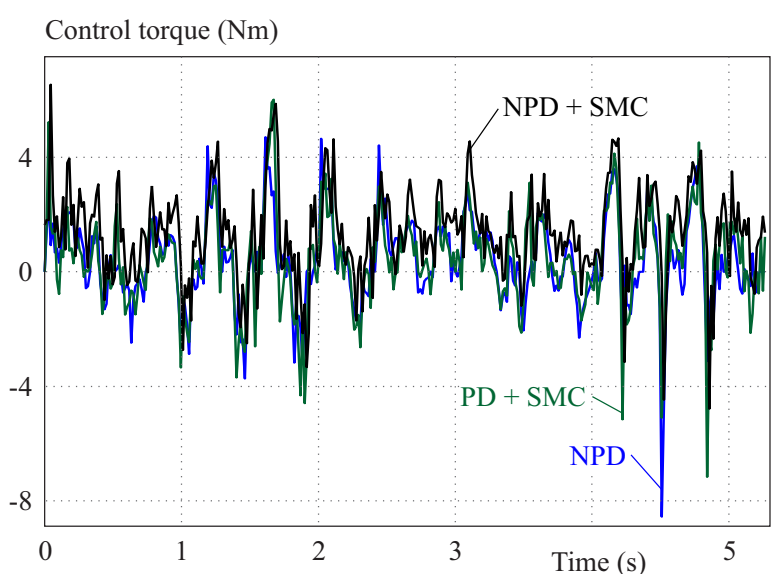

Fig. 13. Experimental torque of joint 2 for case 2

and a maximum acceleration of $15 \mathrm{~m} / \mathrm{s}^{2}$. In order to show the effectiveness and the tracking performances of the proposed approach, the NPD-SMC (10), the NPD and the PD-SMC are implemented.

Figure 2 presents the trajectory tracking in the operational space. Figures $3-5$ depict the tracking error in the direction $x, y$ and $z$ under the proposed NPD-SMC, the NPD proposed in [1], and the PD-SMC proposed in [11]. It is obvious that the proposed controller has better track- 
Table 3. Tracking performance for case 2

\begin{tabular}{lccc}
\hline Controller & NPD [1] & PD+SMC [11] & NPD+SMC \\
\hline$R M S E_{x}(\mathrm{~m})$ & $1.4292 \times 10^{-3}$ & $2.3538 \times 10^{-3}$ & $1.2946 \times 10^{-3}$ \\
$R M S E_{y}(\mathrm{~m})$ & $3.0272 \times 10^{-4}$ & $3.6037 \times 10^{-4}$ & $2.1189 \times 10^{-4}$ \\
$R M S E_{z}(\mathrm{~m})$ & $5.5946 \times 10^{-4}$ & $6.6297 \times 10^{-4}$ & $4.6069 \times 10^{-4}$ \\
$R M S E(\mathrm{~m})$ & $1.5643 \times 10^{-3}$ & $2.4718 \times 10^{-3}$ & $1.3903 \times 10^{-3}$ \\
\hline
\end{tabular}

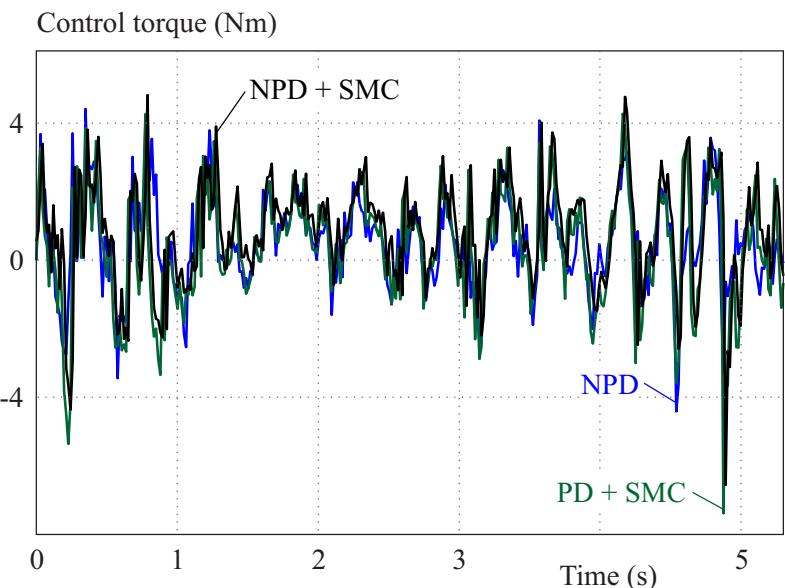

Fig. 14. Experimental torque of joint 3 for case 2

ing error than the other two controllers. More precisely, the NPD-SMC improved the RMSE around $16 \%$ compared to the NPD and $33 \%$ compared to the PD-SMC. On the other hand, the torques of the three controllers have similar variation and amplitude as it can be seen in Figs. 6-8. Table 2 shows the RMSE results of the trajectory tracking experiment of the NPD-SMC, NPD, and PD-SMC.

Case2. In order to study the efficiency of the controller at high cadence movement, a similar experiment is carried out where the same trajectory used before will be executed with a maximum velocity of $10 \mathrm{~m} / \mathrm{s}$ and a maximal acceleration of $20 \mathrm{~m} / \mathrm{s}^{2}$.

The results of the experiment are presented from Fig. 9 to 14. It is clear that the proposed controller still able to provide better performances and decrease the tracking errors during the whole motion process, where the NPDSMC improves the RMSE around $11 \%$ compared to the NPD and $44 \%$ compared to the PD-SMC. Moreover, the control torque stays at an admissible value. Table 3 summarises the performance results, at high dynamic movement. One can conclude that at high dynamic movement the performance improvement is more obvious compared to the PD-SMC, and less obvious compared to the NPD.

\section{Conclusion}

In this work, a NPD-SMC has been proposed for the trajectory tracking of robot manipulators. In order to increase PD control convergence rate, the proportional and derivative gains have been tuned on-line using the tracking error and its time derivative respectively. Also, to benefit from the SMC robustness, only the Signum function has been introduced. The main advantage of the NPD-SMC is its effectiveness and easy implementation that make it highly suitable for industrial applications. Asymptotic convergence of the tracking error and the tracking error rate has been proved using Lyapunov method. Experiments have been carried out on a parallel Delta robot under an accelerations of $15 \mathrm{~m} / \mathrm{s}^{2}$ and $20 \mathrm{~m} / \mathrm{s}^{2}$. It is shown that the proposed approach can obtain superior performance by comparing it with the NPD controller and the PD-SMC controller. On the other hand, the control torques have similar amplitude and variation.

\section{Acknowledgment}

This work was supported in part by Laboratoire de Commande des Processus (LCP), Ecole Nationale Polytechnique, Algiers, and in part by the Algerian Ministry of High Education.

\section{REFERENCES}

[1] W. W.Shang, S. Cong, Z. X. Li and S. L. Jiang, "Augmented Nonlinear PD Controller for a Redundantly Actuated Parallel Manipulator", Advanced Robotics, vol.23, no.12-13, pp.1725-1742, 2009.

[2] W. W. Shang and S. Cong, "Nonlinear computed torque control for a highspeed planar parallel manipulator", Mechatronics vol. 1, no.6, pp.987-992, 2009

[3] C. E. Boudjedir, D. Boukhetala and M. Bouri, "Fuzzy logic iterative learning control for trajectory tracking of parallel kinematic manipulators", 5th International conference on electrical engineering, Algeria, pp 1-6, doi: 10.1109/ICEE-B.2017.8192041, 2017.

[4] F. Haouari, N. Bali, M. S. Boucherit and M. Tadjine, "A Coefficient diagram method controller with backstepping methodology for robotic manipulators", Journal of electrical engineering, vol.66, no.5, pp.270-276, 2015.

[5] M. S. Khireddine and A. Boutarfa, "Reconfigurable control for a Scara robot using RBF networks", Journal of electrical engineering, vol.61, no.2, pp.100-106, 2010.

[6] M. Bennehar, A. Chemori, M. Bouri, L. F. Jenni and F. Pierrot, "A new RISE-based adaptive control of PKMs: design, stability analysis and experiments", International Journal of Control 1-15, 2017.

[7] J. Cerkala and A. Jadlovska, "Application of neural models as controllers in mobile robot velocity control loop", Journal of Electrical Engineering, vol.68, no.1, pp.39-46, 2017. 
[8] X. Lu and M. Liu, "Optimal Design and Tuning of PID-type Interval Type-2 Fuzzy Logic Controllers for Delta Parallel Robots", International Journal of Advanced Robotic Systems , Vol.13, no.3, DOI: 10.5772/63941, 2016.

[9] K. J. Lee, J. J. Choi and J. S. Kim, "A proportional-derivativesliding mode hybrid control scheme for a robot manipulator", Proceedings of the Institution of Mechanical Engineers, PartI: Journal of Systems Control Engineering 218 (8):667-74, 2004.

[10] T. C. Kuo, Y. J. Huang, C. Y. Chen and C. H. Chang, "Adaptive sliding mode control with PID tuning for uncertain systems", Engineering Letters 16(3): 311-315, 2008.

[11] P. R. Ouyang, J. Acob and V. Pano, "PD with sliding mode control for trajectory tracking of robotic system", , Robotics and Computer-Integrated Manufacturing 30 (2014)189-200.

[12] R. Clavel, "Device for movement and displacing of an element in space", US Patent no 4976582, date of patent: December 11, 1990.

[13] A. Codourey, "Dynamic modelling and mass matrix evaluation of the delta parallel robot for axes decoupling control", International Conference on Intelligent Robots and Systems, vol.3, pp.1211-1218, 1996.

[14] M. Rachedi, B. Hemici and M. Bouri, "Design of an H1 controller for the Delta robot: experimental results", Advanced Robotics, vol.29, no.18, pp 1165-1181, 2015.

[15] L. A. Castañeda, A. L. Juárez and I. Chairez, "Robust trajectory tracking of a delta robot through adaptive active disturbance rejection control", IEEE Transactions on control systems technology, vol.23, no.4, pp 1387-1398, 2015.

[16] R. Kelly, V. Santibáñez and A. Loría, Control of Robot Manipulators in Joint Space, Advanced textbooks in control and signal processing, Springer, Berlin, 2005.

[17] F. X. Wu, W. J. Zhang, Q. Li and P. R. Ouyang, "Integrated Design and PD Control of High-Speed Closed-loop Mechanisms", J. Dyn. Sys. Meas. Control 24(4), pp 522-528, 2002.

[18] F. L. Lewis, D. M. Dawson and C. T. Abdallah, Robot Manipulator Control: Theory and Practice, Marcel Dekker, New York (2003).

[19] Z. S. Houa Q. X. Yua and R. H. Chib, "Adaptive Iterative Learning Control for Nonlinear Uncertain Systems with Both State and Input Constraints", Journal of the Franklin Institute, Vol.353, no.15, pp. 2279-2289, 2016.

[20] F. Bouakrif and M. Zasadzinski, "Trajectory tracking control for perturbed robot manipulators using iterative learning method",
Int. J. Adv. Manuf. Technol., doi:10.1007/ s00170-016-8550-3, 2016

21] J. Huang, S. Ri, L. Liu, Y. Wang, J. Kim and G. Pak, "Nonlinear Disturbance Observer-Based Dynamic Surface Control of Mobile Wheeled Inverted Pendulum", IEEE Transactions on Control Systems Technology, vol. 23, no.6, pp.2400-2407, Nov.2015.

[22] J. Slotine and W. Li, Applied nonlinear control, Englewood Cliffs (NJ), Prentice Hall, 1991.

Received 29 July 2018

Chems Eddine Boudjedir was born in Annaba, Algeria in 1991. He received the engineer degree in Automatic Control from Ecole Nationale Polytechnique of Algeria in 2015. He is pursuing his $\mathrm{PhD}$ in the Automatic department of Ecole Nationale Polytechnique (ENP), Algiers, Algeria. His research interests include iterative learning control, robotics, and sliding mode control.

Djamel Boukhetala is a Professor in Automatic Control in the Department of Electrical Engineering of the Ecole Nationale Polytechnique of Algiers. From 1996 to 1999, he was the Head of the Automatic Control Department and the Director of the Laboratoire de Commande des Processus, currently he is the director of post-graduation. His research interests are decentralised control, nonlinear control, fuzzy control and artificial neural networks control applied to robotics, aerospace systems, active suspension and power systems

Mohamed Bouri graduated in Electrical Engineering in 1992 and obtained his PhD degree in 1997 in Industrial Automation at INSA LYON, France. He joined EPFL, Ecole Polytechnique Federal de Lausanne in 1997 and since 2005, he is a group leader of Rehabilitation and Assistive Robotics in the LSRO and lecturer of Robotics and Industrial Robotics. $\mathrm{He}$ is mainly active in the field of robot control, automation and robot design for medical and industrial applications. He has strong references with the development of many industrialized robots (Hita machine (Willemein Macodel), Delta Direct Drive (Bosch Packaging Technology), MotionMaker and WalkTrainer (Swortec), 5 Axis Polishing Delta (Unitechnnologies). 